\title{
Leptophlebiidae (Ephemeroptera) da Amazônia Brasileira
}

\author{
Maria José do Nascimento LOPES ${ }^{1}$, José Moacir Ferreira RIBEIRO², Douglas Fernando PEIRÓ
}

\section{RESUMO}

$\mathrm{Na}$ Amazônia brasileira são registradas dezenove espécies de Leptophlebiidae, distribuídas em dez gêneros. Neste trabalho é apresentada uma lista de todos os gêneros de Leptophlebiidae ocorrentes na Região Neotropical, o numero de espécies de cada gênero no Brasil e um catálogo das espécies registradas na Amazônia brasileira.

\section{PALAVRAS-CHAVE}

Catálogo, Ephemeroptera, Leptophlebiidae, Atalophlebiinae.

\section{Leptophlebiidae (Ephemeroptera) from Brazilian Amazonia}

ABSTRACT

Brazilian Amazonia has nineteen registered species of Leptophlebiidae, distributed in ten genera. In this work a list of all genera of Leptophlebiidae from the Neotropical Region is presented, the number of species of each genus in Brazil and a catalog of the species occurring in the Brazilian Amazon.

KEY-WORDS

Catalog, Ephemeroptera, Leptophlebiidae, Atalophlebiinae.

\footnotetext{
1 Pesquisador INPA/CPEN, Manaus, AM, Brasil, ferreira@inpa.gov.br. fone: (92) 36433205.

2 Bolsista CNPq/INPA, Manaus, AM, Brasil, moacir@inpa.gov.br

${ }^{3}$ Bolsista CAPES, INPA/DCEn, Manaus, AM, Brasil, douglaspeiro@hotmail.com

Financiamento: CNPq/Edital Universal BioArthro; INPA/PPI 3580; 0605.
} 


\section{INTRODUÇÃO}

Os Ephemeroptera são os únicos insetos a apresentarem dois estágios alados, o subadulto (subimago) e o adulto (imago) (Brittain, 1982). Como paleópteros, apresentam uma articulação alar primitiva, que lhes impede de dobrar as asas sobre o abdome quando em repouso (Riek, 1979). As ninfas são aquáticas, a forma do corpo difere totalmente da encontrada no adulto (de vida aérea), possuem tegumento mole e coloração geral do corpo pouco vistosa.

A família Leptophlebiidae Banks, 1900 compreende três subfamílias: Leptophlebiinae Banks, 1900; Atalophlebiinae Peters 1980 e Habrophlebiinae Kluge, 1994. A subfamília Atalophlebiinae é de origem Gondwânica e alcança sua máxima diversidade e abundância em cursos d'água do Hemisfério Sul; as subfamílias Leptophlebiinae e Habrophlebiinae predominam em riachos do Hemisfério Norte (Savage, 1987; Savage et al., 2005). Nenhum membro da subfamília Leptophlebiinae dispersou da América do Norte para a América Central ou para a América do Sul. Os Atalophlebiinae na Região Neotropical estão representados por 49 gêneros sendo que para a maioria destes há poucas espécies descritas.

Leptophlebiidae é uma das famílias de Ephemeroptera mais diversas do país, com 20 gêneros e 48 espécies registradas (Tabela 1); sendo que Miroculis Edmunds, 1963 e Thraulodes Ulmer, 1920 representam os gêneros com maior número de espécies descritas (Peters \& Edmunds, 1972; Savage e Peters, 1983; Domínguez et al., 2002; Salles et al., 2004).

Para a Regiāo Norte alguns trabalhos têm sido realizados, como exemplo citamos a revisão de três gêneros de Leptophlebiidae por Savage \& Peters (1983); a revisão do gênero Fittkaulus Savage e Peters, 1978, por Savage (1986); um estudo sobre a fauna dos escudos das Guianas e brasileiro (em Rondônia), por Lopes (1999); o registro de ocorrência de Ulmeritoides misionensis Domínguez, 1995, para Rondônia, por Da-Silva e Lopes (2001), e a descrição de uma espécie nova de Ulmeritoides Traver, 1959, por Lopes et al. (2003). Vale ressaltar a existência dos trabalhos de Domínguez et al. (2002) sobre o estado do conhecimento da ordem na Região Amazônica; a listagem das espécies de Ephemeroptera do Brasil de Salles et al. (2004) e o site administrado pelo Dr. Frederico Salles http:// ephemeroptera.br/google/epages.com, disponível via internet. Catálogos e listas sobre a família Leptophlebiidae exclusivamente da Amazônia brasileira são inexistentes.

Para que se compreenda melhor a diversidade dessa família é necessário dar continuidade aos estudos sistemáticos em desenvolvimento, bem como intensificar o processo de criação dos imaturos, relacionando-os com os adultos, desse modo resolvendo problemas taxonômicos bem como sinonímias duvidosas. O presente estudo teve como objetivo reunir os dados sistemáticos sobre a família Leptophlebiidae para a Região Neotropical e em particular para a Amazônia brasileira. Ainda, a apresentação de uma lista, em ordem alfabética, dos gêneros ocorrentes na Região Neotropical, o número de espécies de cada gênero registrado até o momento para o Brasil e um catálogo das espécies reportadas para a Amazônia brasileira.

\section{MATERIAL E MÉTODOS}

Neste trabalho é apresentada uma lista, em ordem alfabética, dos gêneros de Leptophlebiidae ocorrentes na Região Neotropical, o número de espécies de cada gênero registrado até o momento para o Brasil e um catálogo das espécies reportadas para a Amazônia brasileira.

A seção do catálogo traz os gêneros, seus autores, ano e espécietipo. As espécies, autor, ano, localidade-tipo (ou locais de ocorrência da espécie), material adicional examinado (quando existente), dados adicionais da coleta e locais de deposição do material. Também são fornecidos os sinônimos (quando existentes). No catálogo a forma adulta (imago) está representada com "I", o subadulto (subimago) com "SI", a ninfa com "N", o macho com o símbolo ơ e, a fêmea com o símbolo 9 .

Os locais de deposição do material são apresentados com as siglas entre parênteses: Florida Agricultural and Mechanic University, Tallashassee, Flórida, Estados Unidos (FAMU); Instituto-Fundación Miguel Lillo, Tucumán, Argentina (IFML); Museu de Zoologia da Universidade de São Paulo, São Paulo, Brasil (MZUSP); National Museum of Natural History, Washington DC, EUA (NMNH); Rijksmuseum van Natuurlijke Historie, Leiden, Holanda (RNH); University of Utah, Utah, Estados Unidos (UU); Zoologische Staatssammlung, Munique, Alemanha (ZSBS); Instituto Nacional de Pesquisas da Amazônia, Manaus, Brasil (INPA); American Museum of Natural History, New York, Estados Unidos (AMNH); Cornell University, New York, Estados Unidos (CUNY); Universidade Federal do Rio de Janeiro, Rio de Janeiro, Brasil (UFRJ); Institut de Recherche pour le Diveloppment, Caiena, Guiana Francesa (ORSTOM).

\section{RESULTADOS}

Na Região Neotropical encontram-se registrados 49 gêneros de Ephemeroptera pertencentes à família Leptophlebiidae. Destes, para o Brasil estão registrados vinte e um gêneros distribuídos em 49 espécies e para a Amazônia brasileira até o momento estão registrados dez gêneros com 19 espécies (Tabela).

\section{CATÁLOGO:}

\section{FAMÍLIA LEPTOPHLEBIIDAE}

\section{GÊNERO Farrodes PETERS}

Farrodes Peters, 1971: 5. Espécie-tipo F. hyalinus Peters (des. orig.).

F. ochraceous Domínguez, Molineri \& Peters, 1996, 94. Holótipo o I, Brasil, Amazonas, Reserva Ducke, km 26. E. 
Manaus, 1-5-ii-1979, O.S. Flint, Jr. (MZUSP).

F. xingu Domínguez, Molineri \& Peters, 1996, 97. Holótipo ơ I, Brasil, Pará, Rio Xingu, Camp. (52 22' W, 3 39' S), Ca $60 \mathrm{~km}$, S. Altamira, 8-16-x-1986. P. Spangler e O.S. Flint (MZUSP). Igarapé Jabuti, armadilha Malaise, dia e noite. Alótipo: I $\%$, mesma data do holótipo (MZUSP). Parátipos:

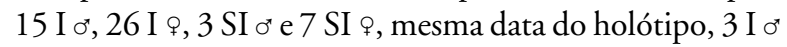
e 3 I q (FAMU e IFML, respectivamente), restante (NMNH).

\section{GÊNERO Fittkaulus SAVAGE \& PETERS}

Fittkaulus Savage \& Peters, 1978: 293. Espécie-tipo F. maculatus Savage \& Peters (des. orig.).

F. maculatus Savage \& Peters 1978: 293. Holótipo I ơ, Brasil, Pará, Igarapé Akahe, Missão Tiriyos, fronteira com BrasilSuriname, 15-iii-1962, E. J. Fittkau. Holótipo (INPA); Alótipo \%, asa em lâmina, mesma data do holótipo; Parátipos $5 \mathrm{~N}$ (INPA) mesma data do holótipo. Material adicional, 1 SI q (criado) e exuvia, Brasil, Pará, Rio Parú, Missão Tiriyos, fronteira Brasil-Suriname, 22-iii-1962, E.J. Fittkau; 1 SI $\odot, 1$ ๑, 1 SI ơ, Brasil, Pará, Igarapé Akahe, missão Tiriyos, fronteira Brasil-Suriname, 15-iii-1962, E.J. Fittkau; 2 N, Brasil, Pará, Igarapé Iveraca I, fronteira Brasil-Suriname, 17-18-iv-1962, E.J. Fittkau (ZSBS).

F. cuiabae Savage, 1986: 265. Holótipo I ๑, Brasil, Mato Grosso, ponte sobre o Rio Aricá, na rodovia Cuiabá/Rondonópolis, perto do $\mathrm{km} 391$. Obtido com armadilha de luz negra, 22-iv1981, D.P. Wojcik; parátipos, 24 I 9, mesma data do holótipo. Holótipo (MZUSP); 20 parátipos (FAMU); 4 parátipos (ZSBS).

F. cururuensis Savage, 1986: 268. Conhecido somente I $q$. Holótipo 1 I q, Brasil, Pará, Rio Cururu, na Missão Cururu, à luz, 6-ii-1961, E.J. Fittkau. Holótipo (MZUSP); parátipo, 1 I ९, mesma data do holótipo (UU).

\section{GÊNERO Hagenulopsis ULMER}

Hagenulopsis Ulmer, 1920. Espécie-tipo H. diptera Ulmer (des.orig.).

F. minuta Spieth, 1943: 1-13. Holótipo I \& (AMNH), Rio Marowijne, Suriname. Outras formas descritas por Peters \& Domínguez, 2001: 353, Brokopondo, Suriname, 27-xii1968, W.L. e J.G. Peters, N; Igarapé Akahe Missão Tiriyos, Fronteira Brasil-Suriname, 15-27-iii-1962, E.J. Fittkau, 2 I ơ, 2 I , SI ơ e SI $q$, parte posterior de SI $q$ e N; N Igarapé Okueima, Fronteira Brasil-Suriname, 18-iv-1962, E.J. Fittkau.

\section{GÊNERO Hermanellopsis DEMOULIN}

Hermanellopsis Demoulin, 1955: 31. Espécie-tipo H. incertans (Spieth) Demoulin, 1955: 31.

Hermanella Spieth, 1943, 1244: 9 (partim); Traver, 1947:
159-160 (partim).

Hermanella (Hermanellopsis) Demoulin 1955: 8.

H. arsia Savage \& Peters, 1983: 108. Holótipo, SI ơ, Brasil, Amazonas, Rio Cuieiras, Norte de Manaus, 24-iv-1961, E.J. Fittkau. Holótipo (INPA); parátipos, 4 SI ơ, da mesma data do holótipo, sendo 2 exemplares (FAMU) e 2 exemplares (UU).

\section{GÊNERO Leentvaaria DEMOULIN}

Leentvaaria Demoulin, 1966: 13. Espécie-tipo L. palpalis Demoulin, 1966: 13.

L. sp. N, Brasil, RR, BR 174, km 914, Rio Paricarana, 28-x1987, Equipe Granfinale. 10 N, sendo 5 (INPA) e 5 (IFML) (Domínguez et al., 2001) todos da mesma data e localidade.

\section{GÊNERO Microphlebia SAVAGE \& PETERS}

Microphlebia Savage \& Peters, 1983: 569. Espécie-tipo, $M$. surinamensis Savage \& Peters (des. orig.).

M. surinamensis Savage \& Peters 1983: 572. Holótipo, SI ơ,: Igarapé Powaka, 200', Suriname, 30-xii-1968, W.L. e J.G. Peters. Parátipos: $1 \mathrm{I} \sigma^{*}$, mesma data do Holótipo; $2 \mathrm{~N}$, mesma localidade do holótipo, 20-xii-1968; 5 N, Igarapé a N.W. of Phedra na rodovia Kraka-Phedra, 200', Brokopondo, Suriname 20-xii-1968, W.L. e J.G. Peters; 1 N, Primeiro igarapé cruzando a rodovia para Hanover, descendo 500$1000 \mathrm{~m}$ a margem do rio, Suriname, 7-viii-1969, lama encharcada na margem do Snog 4, N. Nieser. Holótipo e as 5 $\mathrm{N}$ parátipos (FAMU). 1 SI ơ e $3 \mathrm{~N}$ parátipos (UU). Espécie presente no limite (fronteira) Brasil-Suriname (Dominguez et al. 2002).

M. pallida Savage \& Peters 1983: 574. Holótipo N, Brasil, Amazonas, Rio Branquinho, perto do Rio Cuieiras, Norte de Manaus, na luz, 19-vii-1961, E.J. Fittkau. Parátipos, 1 SI ơ; Rio Branquinho, perto do Rio Cuieiras, Norte de Manaus, Amazonas, na luz, 22-iv-1961, E.J. Fittkau, e 4 N, Igarapé Aracu, $15 \mathrm{~km}$ de Manaus, estrada para Rio Branco, Amazonas 8-vi-1962, E.J.Fittkau. Holótipo e $2 \mathrm{~N}$ parátipos (INPA); 1 SI ơ e $2 \mathrm{~N}$ parátipos (FAMU).

\section{GÊNERO Miroculis EDMUNDS, 1963}

Miroculis Edmunds, 1963: 34. Espécie-tipo, M. (Miroculis) rossi Edmunds, 1963.

\section{SUBGÊNERO Miroculis S.S. EDMUNDS, 1963.}

M.(M.) marauiae Savage \& Peters 1983: 517. Holótipo, I ơ, Brasil, Amazonas, Rio Marauia, Missão S. Antonio, N.W. de Tapuruquara 28-i-1963, E.J. Fittkau. Alótipo, I q , mesmos dados do holótipo. Parátipos, $43 \mathrm{I}$ ơ, mesmos dados do holótipo; 3 I ơ, mesma localidade do holótipo, 25-i-1963; 1 SI ơ e 12 N, mesma localidade do holótipo, 24.i.1963; 2 N, 
igarapé de montanha II, perto do Rio Marauia, 3 dias de viagem acima da Missão S. Antonio, N.W. de Taparuquara, Amazonas, 26-27-i-1963, E.J.Fittkau; 1 N, Igarapé S. Antonio, perto da missão Santo Antonio, Rio Marauia, N.W. de Paparuquara, Amazonas, 9-i-1963, E.J.Fittkau. O holótipo, alótipo (8 I ơ) e parátipos (e $3 \mathrm{~N}$ ) (INPA). Parátipos: 18 I ơ, 1 SI ơ e 6 N (FAMU); 14 I ơ e 3 N, (UU); 6 I ơ e 3 $\mathrm{N}(\mathrm{ZSBS})$.

M.(M.) fittkaui Savage \& Peters, 1983: 529. Holótipo, I ơ, Brasil, Pará, Igarapé Akahe, perto da Missão Tiriyos, próximo da fronteira Brasil-Suriname, 15-iii-1962, E.J. Fittkau. Alótipo, 1 I 9 , mesmos dados do holótipo. Parátipos, 29 I ơ, 20 I $9,13 \mathrm{~N}$, mesmos dados do holótipo; 25 I q, Igarapé Morneni, perto da Missão Tiriyos, próximo da fronteira BrasilSuriname, Pará, 30-iii-1962, E.J. Fittkau. Holótipo, alótipo e parátipos: $5 \mathrm{I}$ ơ, 8 I q e $3 \mathrm{~N}$ (INPA); parátipos: 12 I ๙ 35 I q e $7 \mathrm{~N}$ (FAMU); parátipos: $9 \mathrm{I}$ ơ, $35 \mathrm{I}$ 甲 f e $5 \mathrm{~N}$ (UU); parátipos: $4 \mathrm{I}$ ơ, 12 I 9 e $3 \mathrm{~N}$ (ZSBS); parátipos: $2 \mathrm{I}$ o e $20 \mathrm{I}$ ( $(\mathrm{RNH})$.

SUBGÊNERO Yaruma SAVAGE E PETERS, 1983: 546.

M.(Y.) wandae Savage \& Peters, 1983: 546. Holótipo SI ơ, Brasil, Amazonas, Rio Marauia, um dia de viagem acima da missão Santo Antônio, N.W. de Taparuquara, na luz, 3-ii1963, E.J. Fittkau (INPA).

M. (Y.) sp. Holótipo I ơ, Brasil, Amazonas, Igarapé Aduja, perto ao Rio Itu, 10-ii-1962, E.J. Fittkau, (FAMU) (Savage \& Peters, 1983).

\section{SUBGÊNERO Atroari SAVAGE E PETERS, 1983, 549.}

M. (A.) duckensis Savage \& Peters, 1983: 554. Holótipo 1 I ơ, Brasil, Amazonas, Igarapé Acará, perto do acampamento, Reserva Ducke, N. de Manaus, à luz, 26-vi-1961, E.J.Fittkau. Parátipos: 1 I ơ e 4 N, Cachoeira Gigante, Reserva Ducke, N. de Manaus, Amazonas, 3-vii-1961, E.J. Fittkau; 2 N, mesma localidade, 1-viii-1961; 6 N, Igarapé Barro Branco, Reserva Ducke, N. de Manaus, Amazonas, 10-v-1961, E.J. Fittkau; 3 N, Igarapé Passarinho, Reserva Ducke, N. de Manaus, Amazonas, 27-vi-1961, E.J.Fittkau; 6 N, Igarapé Bica, perto da Ponta Negra, Rio Negro, N. de Manaus, Amazonas, 23vi-1961; 1 N, Cachoeira Gigante, perto da Ponta Negra, Rio Negro, N. de Manaus, Amazonas, 23-vi-1961, E.J. Fittkau. Holótipo (INPA). Parátipos: 4 N (INPA); 1 I ơ e 9 N (FAMU); $9 \mathrm{~N}(\mathrm{UU})$.

M. (A.) amazonicus Savage \& Peters, 1983: 556. Holótipo I ơ, Brasil, Amazonas, Rio Marauia, perto da missão S. Antonio, N.W. de Taparuquara, à luz, 10-i-1963, E.J.Fittkau. Holótipo (INPA). Parátipos, $1 \mathrm{~N}$ \& e $3 \mathrm{~N}$ ơ abaixo da queda d'água, Cachoeira S. Antonio, perto da Missão S. Antonio, N.W. de Taparuquara, Amazonas, 9-i-1963, E.J.Fittkau. Parátipos: 1 $\mathrm{N}$ 甲 e $1 \mathrm{~N}$ ơ \& (FAMU); $2 \mathrm{~N}$ ơ (UU).

\section{GÊNERO Paramaka SAVAGE E DOMINGUEZ}

Paramaka Savage \& Dominguez, 1992: 244. Espécie-tipo $P$. convexa (Spieth) 1943: 10, que é uma combinação de Thraulus convexus Spieth, 1943 com o gênero Paramaka de Savage \& Domínguez, 1992.

P. convexa (Spieth). Combinação estabelecida por Savage \& Domínguez, 1992: 245. Holótipo I ơ, Suriname, Gran Socla, na junção dos rios Litani com o Lawa, 9-vii-1939, D.C. Geijskes. Holótipo (AMNH); e Parátipo, 1 I ơ, alfinetado, mesma data do holótipo. Material adicional do Brasil: Pará, Rio Xingú, camp (52²2’W; 3³9'S) ca 60 km de S. Altamira, Igarapé Jabuti, 8-16-x-1986, P. Spangler e O.S. Flint, Jr. (AMNH).

Thraulus convexus Spieth, 1943: 10. O holótipo é o material do Suriname citado para P. convexa.

Homothraulus convexus, Traver, 1960: 73. O gênero foi criado com base em T. convexus Spieth.

\section{GÊNERO Simothraulopsis DEMOULIN}

Simothraulopsis Demoulin, 1966: 15. Espécie-tipo S. demerara (Traver), 1947, que é uma combinação de Thraulus demerara Traver, 1947 com o gênero Simothraulopsis de Demoulin, 1966 estabelecida por Domínguez, Peters, Peters, Savage, 1997.

S. demerara (Traver). Combinação estabelecida por Domínguez, Peters, Peters, Savage, 1997. Holótipo N, Guiana Inglesa, Mackenzie, Rio Demerara, C.U. 24-vi-1927. Alótipo, uma $\mathrm{N} \&$ do Suriname, Kwakoegron, rio Saramacca, C.U. Entomological Expedition, 12-vi-1937, e parátipos da mesma data do holótipo. Material adicional: Suriname, Distrito Brokopondo, igarapé lado N de Brokopondo, 26/27-xii1968, W.L. \& J. Peters (3 SI ơ e 2 SI o); 16-iv-1975, Messer col. (4 SI ơ e 3 SI ९); Venezuela, Bo. Morichal Tauca, 22 mi, E. Rio Caura, 8-9-ii-1976, C.M. \& O.S. Flint, Jr.. Guiana Francesa, Rio Sinnamary, V. Horeau, col., Saut Dalles, 8-iii1992, 17/18-vii-1992 (3 SI क), 25-vii-1993 (3 SI क), 8-iii1992 (6 SI ๑), 10-v-1994 (1 SI ơ), 7-ii-1994; Saut Maipouri, 24/26-v-1993 (8 SI 甲), 23-xi-1993 (1 SI ơ); Saut Aimara, 14-vi-1994 (1 SI ॰); No 1817 piége. lum. (2 ơ e 7 SI ॰); Saut Deux Roros, 1-xi-1992 (24 N). Parátipos da Amazônia Brasileira: Pará, Rio Paru, cachoeira abaixo da Maloca Tiriyo, 20-iii-1961, W. Sattler (1 ơ); Rio Branco, Boa Vista, 17-ix1941, H. Sioli (1 N); Amazonas, Rio Aripuana, Beneficente, 18-i-1962, à luz, E.J. Fittkau; Amazonas, à luz em R/V Alpha Helix, S. Antonio do Iça, Rio Solimões, 22-ii-1977, J.B.Wallace. Registro de 35 localidades no Brasil coletadas por E.J. Fittkau entre ii-1961 e ii-1963, do Pará, na área do Rio Parú e Rio Cururú; e Amazonas, Rio Negro e tributários acima de Manaus, particularmente no Rio Cuieiras e Rio Maurauia. Material depositado no CUNY, FAMU, NMNH, 
Tabela 1 - Gêneros de Leptophlebiidae (Ephemeroptera) na Região Neotropical, número de espécies registradas para o Brasil e Amazônia brasileira.

\begin{tabular}{|c|c|c|c|}
\hline $\begin{array}{c}\text { Gêneros da Região } \\
\text { Neotropical }\end{array}$ & Subgênero & $\begin{array}{c}\text { Número de espécies } \\
\text { para o Brasil }\end{array}$ & $\begin{array}{l}\text { Número de espécies para } \\
\text { a Amazônia Brasileira }\end{array}$ \\
\hline Archethraulodes & & 0 & 0 \\
\hline Askola & & 1 & 0 \\
\hline Atalonella & & 0 & 0 \\
\hline Atalophlebioides & & 0 & 0 \\
\hline Atopophlebia & & 0 & 0 \\
\hline Bessierus & & 0 & 0 \\
\hline Borinquena & & 0 & 0 \\
\hline Careospina & & 0 & 0 \\
\hline Choroterpes & & 0 & 0 \\
\hline Dactylophlebia & & 0 & 0 \\
\hline Demoulinellus & & 0 & 0 \\
\hline Ecuaphlebia & & 0 & 0 \\
\hline Farrodes & & 3 & 2 \\
\hline Fittkaulus & & 3 & 3 \\
\hline Gonserellus & & 0 & 0 \\
\hline Hagenulopsis & & 2 & 0 \\
\hline Hagenulus & & 0 & 0 \\
\hline Hapsiphlebia & & 0 & 0 \\
\hline Hermanella & Hermanella & 1 & 0 \\
\hline$H$. & Guayakia & 3 & 0 \\
\hline Hermanellopsis & & 1 & 1 \\
\hline Homothraulus & & 0 & 0 \\
\hline Hydrosmilodon & & 0 & 0 \\
\hline Hylister & & 1 & 0 \\
\hline Leentvaaria & & 1 & 1 \\
\hline Lisetta & & 0 & 0 \\
\hline Magallanella & & 0 & 0 \\
\hline Massartella & & 3 & 0 \\
\hline Massartellopsis & & 0 & 0 \\
\hline Meridialaris & & 0 & 0 \\
\hline Microphlebia & & 1 & 1 \\
\hline Miroculis & Miroculis & 3 & 2 \\
\hline M. & Yaruma & 2 & 2 \\
\hline M. & Atroari & 2 & 2 \\
\hline M. & Ommaethus & 2 & 0 \\
\hline Miroculitus & & 0 & 0 \\
\hline Needhamella & & 1 & 0 \\
\hline Neohagenulus & & 0 & 0 \\
\hline Nousia & & 0 & 0 \\
\hline Paramaka & & 1 & 1 \\
\hline Penaphlebia & Megalophlebia & 0 & 0 \\
\hline$P$ & Penaphlebia & 0 & 0 \\
\hline Perissophlebiodes & & 1 & 0 \\
\hline Rhigotopus & & 0 & 0 \\
\hline
\end{tabular}

\begin{tabular}{|c|c|c|c|}
\hline $\begin{array}{l}\text { Gêneros da Região } \\
\text { Neotropical } \\
\end{array}$ & Subgênero & $\begin{array}{c}\text { Número de espécies } \\
\text { para o Brasil }\end{array}$ & $\begin{array}{c}\text { Número de espécies para } \\
\text { a Amazônia Brasileira }\end{array}$ \\
\hline Secochela & & 0 & 0 \\
\hline Segesta & & 1 & 0 \\
\hline Simothraulopsis & & 1 & 1 \\
\hline Terpides & & 0 & 0 \\
\hline Thraulodes & & 7 & 0 \\
\hline Tikuna & & 1 & 1 \\
\hline Traverella & & 1 & 0 \\
\hline Traverina & & 0 & 0 \\
\hline Ulmeritoides & & 4 & 2 \\
\hline Ulmeritus & & 2 & 0 \\
\hline Totais & & 49 & 19 \\
\hline
\end{tabular}

ORSTOM, IFML e ZSBS.

Thraulus demerara Traver, 1947: 150; 1960: 73 . O holótipo é o material do Suriname.

Simothraulopsis surinamensis Demoulin, 1966: 15.

Simothraulopsis demerara (Traver). Combinação estabelecida por estabelecida por Domínguez, Peters, Peters, Savage, 1997.

\section{GÊNERO Tikuna SAVAGE, FLOWERS \& PORRAS}

Tikuna Savage, Flowers \& Peters, 2005: 2. Espécie-tipo Choroterpes atramentum Traver, 1947: 156.

T. bilineata (Needham \& Murphy). Combinação estabelecida por Peters, Flowers, Hubbard, Domínguez \& Savage, 2005, com base em Choroterpes bilineata proveniente de La Chorrera, Putumayo Dist, Peru. Material adicional: Suriname, Wijne Dist., Moengo, Boven, 1/28-v-1927, P.P Babiy (1 SI ơ); Venezuela, Zulia State, Dist. Mara, Río Socuy, Campamento Corpozulia, 50 km W de Carrasquero, 6/7-x-1979, H.M. Savage \& R.A. Romero (1 SI ণ̛); Perija El Tucuco, Mission El Tucuco Río El Tucuco, $1 / 2 \mathrm{~km}$ da igreja, 1/5-x-1979, H.M..Savage (1 I \&) ; Equador, Pastaza Prov., Tzapino, 32 km NE Tigueno, 111' S, 77²14' W, 400m, 25-v-1976, J. Cohen, Coll (37 I ९); Limoncocha (70 miles SE), 30-v-1976, J. Cohen, Coll (2 I क); Cononaco, 3 FI, 30-v-1976; Amazônia Brasileira, Pará, Rio Cururu, 100 km acima da Missão Cururu, a luz, 25-I-1962, E,J. Fittkau. Material depositado no CUNY, FAMU

Choroterpes bilineata Needham \& Murphy, 1924; Traver, 1947: 156. O holótipo é o material do Peru.

\section{GÊNERO Ulmeritoides TRAVER}

Ulmeritoides Traver, 1959: 8 (como subgênero). Espécie-tipo Ulmeritus (Ulmeritoides) uruguaiensis Traver, (1959: 8). Ulmeritoides foi estabelecido como um subgênero de Ulmeritus 
por Traver, 1959, baseado em indivíduos adultos. Em 1991 Dominguez elevou o subgênero ao status genérico.

U. misionensis Domínguez, 1995: 25. Holótipo I ơ, Argentina, Misiones, Inta San Vicente, 30-xi-1986, E. Domínguez. Holótipo (IFML). Parátipos: 1 I ơ (IFML), 1 SI ơ (IFML), 3 SI 9 (IFML), $20 \mathrm{~N}$ (IFML), $10 \mathrm{~N}$ (FAMU) e $10 \mathrm{~N}$ (NMNH), todos do mesmo local e data do holótipo. A espécie foi observada pela primeira vez no Brasil, baseado em quatro ninfas do Estado de Rondônia, Região Amazônica: 1 N ơ e 1 $\mathrm{N}$ \%, encontrada na foz do Rio Candeias (10³0'S; 615'W), em 14-viii-1985 (UFRJ), e $2 \mathrm{~N}$ q do Igarapé Ribeirão (10¹5'S, 61'' 120 m), 09-viii-1985 (INPA). Esta é a terceira espécie nominal de Ulmeritoides que ocorre no Brasil, após U. uruguayensis, (Traver, 1959) e U. patagiatus, (Thew, 1960), ocorrendo no Estado de Santa Catarina.

U. oepa Lopes, Da-Silva \& Py-Daniel, 2003: 195. Holótipo: 1 N ơ (INPA). Localidade-tipo: Brasil, Roraima, Rio Uraricoera (0327’N, 6058’W, 90 m), Igarapé Xiquibá, 14-x-1987, Py-Daniel, Dellome, Sandra, Coscaron e Ulisses. Parátipos: 2 $\mathrm{N}$ ơ $3 \mathrm{~N}$ 甲 mesma data do holótipo; nove ninfas (seis machos e quatro fêmeas), Rio Uraricoera (tributário do rio Araça), Igarapé Vermelho, 12-vii-1987, Py-Daniel. Doze parátipos (três do Igarapé Xiquibá e nove do Igarapé Vermelho) depositados INPA, dois parátipos (um macho e uma fêmea do Igarapé Xiquibá) na UFRJ.

\section{AGRADECIMENTOS}

Aos revisores anônimos, pelas valiosas sugestões fornecidas quando da revisão ao manuscrito.

\section{BIBLIOGRAFIA CITADA}

Brittain, J.E. 1982. Biology of Mayflies. Ann. Rev. Entomol., 27: 119-147.

Da-Silva, E.R.; Lopes, M.J.N. 2001. First record of Ulmeritoides misionensis (Ephemeroptera: Leptophlebiidae) in Brazil. Rev. Biol. Trop., 49: 3 .

Demoulin, G. 1955. Une mission Belge au Brésil, Éphéméroptères. Bull. Inst. R. Sci. Nat. Belg., 31(20):1-32.

Demoulim, G. 1966. Contribution a l'etude des Ephemeropteres du Surinam. Bull. Inst. R. Sci. Nat. Belg., 42(37): 1-22.

Domínguez, E. 1991. The status of the genus Ulmeritus (Ephemeroptera: Leptophlebiidae: Atalophlebiinae) and related taxa. In: AlbaTercedor J. \& Sanchez-Ortega (Eds). Overview and Strategies of Ephemeroptera and Plecoptera. Sandhill Crane Press, Gainesville, Florida, p. 157-167.

Domínguez, E. 1995. Cladistic analysis of the Ulmeritus-Ulmeritoides group (Ephemeroptera, Leptophlebiidae), with descriptions of five new species of Ulmeritoides. J. New York Entomol. Soc., 103 (1): 15-38.

Domínguez, E.; Ferreira, M.J.; Nieto, C. 2001. Redescription and phylogenetic relationship of Leentvaaria Demoulin
(Ephemeroptera: Leptophlebiidae). In: Domínguez, E. (Ed). Trends in Research in Ephemeroptera \& Plecoptera. Kluger Academic/Plenum Publishers, New York, Estados Unidos, p. 313-320.

Domínguez, E.; Molineri, C.; Peters, W.L. 1996. Ephemeroptera from Central and South America: New species of the Farrodes bimaculatus Group with a key for the males. Stud Neotropica Fauna Environ., 31: 87-101.

Domínguez, E.; Peters, W.L.; Peters, J.; Savage, H.M. 1997. The imago of Simothraulopsis Demoulin with a redescription of the nymph (Ephemeroptera: Leptophlebiidae: Atalophlebiinae). Aquatic Insects, 19(3): 141-150.

Domínguez E.; Zuñiga, M.C.; Molineri, C. 2002. Estado actual del conocimiento y distribución del Orden Ephemeroptera (Insecta) en la Región Amazónica. Caldasia, 24 (2): 459-469.

Edmunds, G.F., Jr. 1963. A new genus and species of mayfly from Peru (Ephemeroptera: Leptophlebiidae). Pan-Pac. Entomol, 39: 34-36.

Edmunds, G.F., Jr.; Jensen S.; Berner. L. 1976. Mayflies of North and Central America. Uni. of Minn. Press, St. Paull, Minn. 330pp.

Lopes, M.J.N. 1999. Sistemática de Atalophlebiinae (Insecta: Ephemeroptera: Leptophlebiidae) dos Escudos das Guianas e Brasileiro (Rondônia). Tese de Doutorado, Instituto Nacional de Pesquisas da Amazônia/Universidade Federal do Amazonas, Manaus, Amazonas. 71pp.

Lopes, M.J.N.; Da-Silva, E.R.; Py-Daniel, V. 2003. A new species of Ulmeritoides from Brazil (Ephemeroptera: Leptophlebiidae). Rev. Biol. Trop., 51(1): 195-200.

Peters, W.L. 1971. A revision of the Leptophlebiidae of West Indies (Ephemeroptera). Smith. Contr. Zool., 62: 1-48.

Peters, W.L.; Domínguez, E. 2001. The identity of Hagenulopsis minuta Spieth (Leptophlebiidae: Atalophebiinae). In: Domínguez, E. (Ed).Trends in Research in Ephemeroptera \& Plecoptera. Kluger Academic/Plenum Publishers, New York, Estados Unidos, p. 353-358.

Peters, W.L.; Edmunds G.F, 1972. A revision of the generic classification of certain Leptophlebiidae from Southern South America (Ephemeroptera). Ann. Ent. Soc. Am., 65(6): 13981414.

Peters, J.G.; Flowers, R.W.; Hubbard, M.D.; Domínguez, E.; Savage, H.M. 2005. New records and combinations for Neotropical Leptophlebiidae (Ephemeroptera). Zootaxa, 1054: 51-60.

Riek, E.F. 1979. Ephemeroptera. In: Sciro. Insects of Australia. Melbourne University Press, Austrália. 1029pp.

Salles, F.F.; Da-Silva, E.R.; Hubbard, M.D.; Serrão, J.E. 2004. As espécies de Ephemeroptera (Insecta) registradas para o Brasil. Biota Neotropica, 4 (2) (http://www.biotaneotropica.org.br/ $\mathrm{v} 4 \mathrm{n} 2 / \mathrm{pt} / \mathrm{abstract}$ ?article+BN02404022004). Acesso 30/06/ 2005.

Savage, H.M 1986. Systematics of the Terpides lineage from the Neotropics: Definition of the Terpides lineage, methods and revision of Fittkaulus. Savage \& Peters. Spixiana, 9 (3): 255-270. 


\section{ACTA}

Savage, H.M. 1987. Biogeographic classification of the Neotropical Leptophlebiidae (Ephemeroptera) based upon geological centers of ancestral origin and ecology. Stud. Neotrop. Fauna Envir., 22 (4): 199-222.

Savage, H.M.; Peters, W.L. 1978. Fittkaulus maculatus a new genus and species from northern Brazil (Leptophlebiidae: Ephemeroptera). Acta Amazonica, 8: 293-298.

Savage, H.M.; Peters, W.L. 1983. Systematics of Miroculis and related genera from Northern South America (Ephemeroptera: Leptophlebiidae). Trans. Amer. Entomol. Soc., 108(4): 491-600.

Savage, H.M.; Dominguez, E. 1992. A new genus of Atalophlebiinae (Ephemeroptera: Leptophlebiidae) from Northern South America. Aquatic Insects, 4: 243-248.

Savage, H.M.; Flowers, R.W.; Porras V., W. 2005. Rediscovery of Choroterpes atramentum in Costa Rica, type species of Tikuna new genus (Ephemeroptera: Leptophlebiidae: Atalophlebiinae), and its role in the "Great American Interchange". Zootaxa, 932: $1-14$.
Spieth, H.T. 1943. Taxonomic studies on the Ephemeroptera. In: III Some interesting ephemerids from Surinam and other Neotropical localities. Am. Mus. Novit. 1244: 1-13.

Thew, T.B. 1960. Taxonomic studies on some Neotropical Leptophlebiid mayflies. Pan Pacif. Ent., 36(3): 119-132.

Traver, J.R. 1947. Notes on Neotropical Mayflies. Part II. Family Baetidae, subfamily Leptophlebiidae. Rev. Entomol. (Rio de Janeiro), 18:149-160.

Traver, J.R. 1959. Uruguayan mayflies. Family Leptophlebiidae. Part I. Rev. Soc. Urug. Ent., 3: 1-13.

Traver, J.R. 1960 Uruguayan mayflies. Family Leptophlebiidae. Part III. Rev. Soc. Urug. Ent., 4: 73-86.

Traver, J.R.; Edmunds, Jr., G.F. 1967. A revision genus Thraulodes (Ephemeroptera: Leptophlebiidae). Miscell. Pub. Entomol. Soc. Amer., 349-395.

Recebido em 06/10/2006

Aceito em 02/02/2007 
